\title{
A comparative analysis of several non-local fracture criteria
}

\author{
L. P. Isupov S. E. Mikhailov
}

Summary Comparative analysis was carried out for three non-local fracture criteria (NLFC) in application to plane problems: the average stress fracture criterion (ASFC), the minimum stress fracture criterion (MSFC) and the fictitious crack fracture criterion (FCFC). Each of them may be considered as a particular form of the general non-local strength functional. All the criteria contain two material parameters: a characteristic length and the tensile strength (ASFC and MSFC) or the critical stress intensity factor (FCFC).

These criteria were used for a strength description of a plate containing a smooth stress concentrator (circular hole) or a singular stress concentrator (central straight crack). It was ascertained that ASFC and FCFC lead to identical results for the symmetrically loaded central straight crack. ASFC and MSFC may be successfully used for the description of strength of the bodies with smooth as well as singular concentrators while the FCFC gives incorrect predictions for large smooth concentrators and for some other cases. Comparison of predicted and experimental data has shown that ASFC is preferable in most cases but nevertheless there exists a systematic deviation of experimental points from the criterion predictions.

Key words fracture mechanics, strength, non-local fracture criteria, comparison with experiment.

\section{1}

\section{Introduction}

In the traditional (local) approach, the strength of a body in an analyzed point $y$ is characterized by the value of some function of stress tensor components at the same point without consideration of the stress state in other points. The local fracture criteria can be represented, e.g., in the form

$$
f\left(\sigma_{i j}(y)\right)=\sigma_{c},
$$

where $f$ is a material function and $\sigma_{c}$ is a material constant. These criteria give a good description of experimental data when the stress distribution is close to a uniform stress state.

\section{P. Isupov}

M.V.Lomonosov Moscow State University, Faculty of Mechanics and Mathematics, 119899 Moscow, Vorob'evy Gory, Russia, e-mail: isupov@nw.math.msu.su;

\section{S. E. Mikhailov}

Wessex Institute of Technology, Ashurst Lodge, Ashurst, Southampton, SO40 7AA, UK,

E-mail:mik@wessex.ac.uk 
There are several problems of strength and fracture mechanics that can not be solved (or are tedious to solve) by use of traditional strength conditions. Such problems include the strength small-scale effects, strength description of bodies with singular stress concentrators (corner points, intersection of interfaces) generating singularities with different exponents, the problem of unification of strength conditions for bodies with smooth and singular concentrators. Some examples of the problems are given in Fig.1-3.

An example of the strength small-scale effect is presented in Fig.1. An infinite elastic plate with a circular hole is considered, which is loaded at the infinity by a uniform traction $q$. It is known from the elasticity theory that the maximum stress is independent of the hole radius $a$, is equal to $3 q$, and is realized in the boundary point $y$. The plate strength evaluated by use of the fracture criterion $\sigma_{\theta \theta}=\sigma_{c}$ is then independent of the hole radius and is equal to one third of the strength $\sigma_{c}$ of the plate without a hole (the solid line). However appropriate fracture test data for plates with small holes (see, e.g., [1-3]) pointed out schematically in the figure show that the plate strength depends actually on the hole radius.

Another example of the strength small-scale effect is delivered by the same plate but now with a crack, having a length $2 a$, instead of a hole, Fig. 2. From the linear elasticity one obtains the value for the stress intensity factor $K_{1}(y)=q \sqrt{\pi a}$. From the linear fracture mechanics one has the fracture criterion $K_{1}(y)=K_{1 c}$, where $K_{1 c}$ is a material constant - fracture toughness. Using these two expressions, we get the theoretical dependence of the plate strength from the crack length (the solid line), according to which the plate strength tends to infinity as the crack length tends to zero. But the experiments for short cracks (see, e.g., $[2,4,5]$ ) show that the strength tends to a finite value.

The same plate, but now with a diamond-shaped hole (Fig. 3), delivers an example of a singular concentrator with a not square root singularity. According to the linear elasticity, we have the following stress behavior near the corner points: $\sigma_{i j}(\rho, \theta) \sim$ $K_{1}(y ; \alpha) \rho^{-\gamma(\alpha)} f_{i j}(\theta ; \alpha)$, where the exponent $\gamma$ depends only on the angle $\alpha$. It is not possible to estimate the strength of a body with such stress behavior either by the traditional local strength condition or by the local linear fracture mechanics condition. In principle, one can try to use the strength condition $K_{1}(y ; \gamma)=K_{1 c}(\gamma)$, which is analogous to the linear fracture mechanics condition. However, one must then determine the critical strength intensity factor $K_{1 c}$ experimentally for each $\gamma$, that is, for each angle $\alpha$, what is rather tedious and expensive. Moreover, the same difficulties occur with the small-scale effect as for short cracks or small circular holes.

These three examples show the necessity of a more general strength theory. Such a theory should describe the small-scale effects and be applicable to bodies without cracks, with cracks as well as with other singular concentrators. These conditions meet the nonlocal strength theories.

A functional approach to non-local strength conditions and fracture criteria was suggested in $[6,7,8]$, where a general form of non-local strength condition based on a nonlinear space strength functional was proposed. The strength functional (functional safety factor) is associated with the supremum of a positive factor by which a given stress field may be multiplied to obtain a non-fracturing stress field.

There are a number of non-local fracture criteria proposed earlier, which can be considered as particular cases of the general strength functional form. The objective of this work is a comparative analysis of some non-local fracture criteria on the basis of experimental data for the bodies containing smooth and singular concentrators. 


\section{2}

\section{Three non-local fracture criteria for the plane problems.}

Three most popular non-local fracture criteria for the two-dimensional case are represented in [6] in some generalized forms. Let $(\rho, \theta)$ be a local polar coordinate system with the center at an analyzed point $y$ of a body; let $\eta(\theta)$ be a unit vector making an angle $\theta$ with the coordinate axis; and let $\sigma_{\rho \rho}, \sigma_{\theta \theta}, \sigma_{\rho \theta}$ be the stress components in this coordinate system.

2.1 Fracture criterion based on average stress over a characteristic length $d_{1}$ (ASFC)

This approach was considered by Neuber [9], Novozhilov [10], Whitney and Nuismer [2] and other authors. It can be written in the following generalized form:

$$
\frac{1}{d_{1}} \max _{-\pi<\theta \leq \pi} \int_{0}^{d_{1}} \sigma_{\theta \theta}(y+\rho \eta(\theta)) d \rho=\sigma_{c} .
$$

Here $\sigma_{c}$ is the strength of a body without concentrators under uniform traction; $d_{1}$ is a material constant with length measure.

If the direction $\theta_{0}$, where the maximum in (1) is realized, is known, the ASFC has the simplest form:

$$
\frac{1}{d_{1}} \int_{0}^{d_{1}} \sigma_{\theta \theta}\left(y+\rho \eta\left(\theta_{0}\right)\right) d \rho=\sigma_{c},
$$

where integration is performed along this direction.

\subsection{Fracture criterion based on a minimum stress over a characteristic length} $d_{2}$ (MSFC)

This approach was used by Whitney and Nuismer [2] and other authors. In a generalized form, this criterion may be written as:

$$
\max _{-\pi<\theta \leq \pi}\left[\min _{0 \leq \rho \leq d_{2}} \sigma_{\theta \theta}(y+\rho \eta(\theta))\right]=\sigma_{c}
$$

Here $\sigma_{c}$ and $d_{2}$ are material constants. In the case of a known direction $\theta_{0}$, where the maximum in (3) is realized, the MSFC may be written in a more simple form:

$$
\min _{0 \leq \rho \leq d_{2}} \sigma_{\theta \theta}\left(y+\rho \eta\left(\theta_{0}\right)\right)=\sigma_{c}
$$

\subsection{Fracture criterion based on a model of fictitious crack with a characteristic length $d_{3}$ (FCFC)}

Criterion of such type was used by Waddoups et al.[1], Cruse [11], Caprino et al.[12] and other authors. It is supposed that there exists a fictitious crack with a characteristic length $d_{3}$ originating from the considered point $y$ of the body. After some modification [6], this criterion may be represented in the following form:

$$
\max _{-\pi<\theta \leq \pi} \min _{i} K_{1 i}\left(y, \theta, d_{3}\right)=K_{1 c} .
$$


Here $K_{1 c}$ and $d_{3}$ are material constants. $K_{11}=K_{1}(y)$ and $K_{12}=K_{1}\left(y+d_{3} \eta(\theta)\right)$ are the stress intensity factors at the ends of the fictitious crack laying along the $\eta(\theta)$ direction.

If the direction $\theta_{0}$ of fracture is considered to be known, this yields from (5):

$$
\min \left(K_{1}(y), K_{1}\left(y+d_{3} \eta\left(\theta_{0}\right)\right)=K_{1 c}\right.
$$

For an edge crack beginning from the body boundary, there exists only one stress intensity factor $K_{1}\left(y+d_{3} \eta\left(\theta_{0}\right)\right)$ and the minimum disappears in (6).

Each of the criteria written above includes two material parameters: a characteristic length $d_{i}$ and the strength parameter $\sigma_{c}$ or $K_{1 c}$. These two parameters can be determined from two independent macro experiments. The following fracture tests may be, for example, chosen: the tensile loading of a smooth specimen without concentrators and tensile loading of the specimen with a transverse crack or with a circular hole.

\section{3}

\section{Strength of the plate containing a central straight crack.}

A body containing a straight central crack provides a convenient object for the analysis of the NLFC validity. Consider a straight crack of a length $2 a$ in a plate of the infinite extent. The origin of the $\left(x_{1}, x_{2}\right)$ coordinate system coincides with the center of the crack. If a uniform tensile traction $\sigma$ is applied parallel to the $x_{2}$ axis at infinity, then $\sigma_{22}\left(x_{1}, 0\right)$ near the crack tip is approximated asymptotically (see, e.g., [13]) by the expression:

$$
\sigma_{22}\left(x_{1}, 0\right)=\frac{K_{1}}{\sqrt{2 \pi\left(x_{1}-a\right)}},
$$

where $K_{1}$ is the mode 1 stress intensity factor given by the expression

$$
K_{1}=\sigma \sqrt{\pi a} .
$$

Equation (7) presents only the main part of the asymptotic decomposition of the stress field near the crack tip and it is usually considered to be quite correct for $(x-a) / a \leq 0.1$. For small cracks, however, the fracture criteria may require an accurate knowledge of the stress distribution near the crack tip. An exact expression for the normal stress ahead of the crack can be obtained, e.g., as a limiting case of the solution for an elliptical hole in an infinite plate $[14, \mathrm{Ch}$. III, Sect. 1]. It should be noted that the result is the same for isotropic as well as for anisotropic plates:

$$
\sigma_{22}\left(x_{1}, 0\right)=\frac{K_{1} x_{1}}{\sqrt{\pi a\left(x_{1}^{2}-a^{2}\right)}} .
$$

The line of maximal tensile stress coincides with the continuation of the crack direction along the $x$ axis and all NLFC given above may be used in the simplest forms (2), (4) and (6).

\subsection{Average stress fracture criterion (ASFC)}

Applying the criterion (2) to the end point of the crack in conjunction with equation (9) yields:

$$
K_{1 c}=\sigma_{c} \sqrt{\pi d_{1} \eta_{1} /\left(1+\eta_{1}\right)}
$$


where $\eta_{1}=a /\left(a+d_{1}\right)$ is the normalized crack length. Let us denote $q=\sigma$ the tensile strength of the plate with crack. Then, from the equations (8) and (10), one can obtain the relation between the normalized plate strength and crack length:

$$
\frac{q}{\sigma_{c}}=\sqrt{\frac{1-\eta_{1}}{1+\eta_{1}}}
$$

It follows from (11), that $q / \sigma_{c} \rightarrow 1$ when $\eta_{1} \rightarrow 0 \quad(a \rightarrow 0)$ for the short crack; and $q / \sigma_{c} \rightarrow 0$ when $\eta_{1} \rightarrow 1 \quad(a \rightarrow \infty)$ for the long crack.

It is interesting to note that the critical stress intensity factor $K_{1 c}$ used generally in the linear fracture mechanics is not a material constant, when a non-local fracture criterion is applied. It is the function of the crack length given by (10).

As $a$ increases, the value $K_{1 c}$ asymptotically approaches the limiting constant value $K_{1 c}^{\infty}$ for large cracks:

$$
K_{1 c}^{\infty}=\sigma_{c} \sqrt{\pi d_{1} / 2}
$$

(This value of $K_{1 c}$ may be obtained at once if we use criterion (2) and stress asymptotics (7) approximating (9) for sufficiently large cracks.) Just this, determined from the long crack tests, value $K_{1 c}^{\infty}$ should be regarded as a material constant from the view point of non-local fracture criteria.

If $\sigma_{c}$ and $K_{1 c}^{\infty}$ are defined from independent experiments, we can express $d_{1}$ from (12):

$$
d_{1}=\frac{2}{\pi}\left(\frac{K_{1 c}^{\infty}}{\sigma_{c}}\right)^{2}
$$

If $d_{1}$ is obtained from a plate strength $q^{\prime}$ experimentally measured for a crack length $2 a^{\prime}$ and from $\sigma_{c}$ also known from a fracture test, then

$$
d_{1}=2 a^{\prime} \frac{\left(q^{\prime} / \sigma_{c}\right)^{2}}{1-\left(q^{\prime} / \sigma_{c}\right)^{2}}
$$

\subsection{Minimum stress fracture criterion (MSFC)}

According to criterion (4) and equation (9), the following relation is valid for the dependence of the critical stress intensity factor on the crack length:

$$
K_{1 c}=\sigma_{c} \sqrt{\pi d_{2} \eta_{2}\left(1+\eta_{2}\right)}
$$

where $\eta_{2}=a /\left(a+d_{2}\right)$. Using (8) for $\sigma=q$ together with (14), one can obtain

$$
q / \sigma_{c}=\sqrt{1-\eta_{2}^{2}}
$$

As it was done in the previous section, the following results can be easily derived:

$$
K_{1 c}^{\infty}=\sigma_{c} \sqrt{2 \pi d_{2}} ; \quad d_{2}=\frac{1}{2 \pi}\left(\frac{K_{1 c}^{\infty}}{\sigma_{c}}\right)^{2}
$$

or

$$
d_{2}=a^{\prime} \frac{1-\sqrt{1-\left(q^{\prime} / \sigma_{c}\right)^{2}}}{\sqrt{1-\left(q^{\prime} / \sigma_{c}\right)^{2}}},
$$

when $d_{2}$ is evaluated from a test for a plate with a finite crack of length $2 a^{\prime}$ and from $\sigma_{c}$. 
Comparison of (13) and (16) gives

$$
d_{2}=\alpha_{2} d_{1}, \quad \alpha_{2}=\frac{1}{2\left[1+1 / \sqrt{1-\left(q^{\prime} / \sigma_{c}\right)^{2}}\right]}=\frac{1}{2\left[1+\sqrt{\left(1+\eta_{1}^{\prime}\right) /\left(2 \eta_{1}^{\prime}\right)}\right]}
$$

where $\eta_{1}^{\prime}:=a^{\prime} /\left(a^{\prime}+d_{1}^{\prime}\right)$. Since $q^{\prime} / \sigma_{c} \rightarrow 0$ when $a \rightarrow \infty$, we have from (17) the relation $d_{1}=4 d_{2}$ for the case when $d_{1}$ and $d_{2}$ are obtained from the critical stress intensity factor $K_{1 c}^{\infty}$ for a long crack and from the longitudinal strength $\sigma_{c}$ of the plate without concentrators.

\subsection{Fictitious crack fracture criterion (FCFC)}

In accordance with this approach, one has to add the fictitious crack of the length $d_{3}$ to the considering end of the main crack of the length $2 a$. The stress intensity factor for this composed crack may be written in the form:

$$
K_{1}=\sigma \sqrt{\pi\left(a+d_{3} / 2\right)}
$$

and criterion (6) gives

$$
K_{1 c}^{\infty}=q \sqrt{\pi\left(a+d_{3} / 2\right)} .
$$

For the plate without crack, we have $\mathrm{a}=0$ and $K_{1 c}^{\infty}=\sigma_{c} \sqrt{\pi d_{3} / 2}$. Thus

$$
d_{3}=\frac{2}{\pi}\left(\frac{K_{1 c}^{\infty}}{\sigma_{c}}\right)^{2}
$$

and

$$
\frac{q}{\sigma_{c}}=\sqrt{\frac{1-\eta_{3}}{1+\eta_{3}}}
$$

where

$$
\eta_{3}=a /\left(a+d_{3}\right)
$$

If $d_{3}$ is evaluated from a test for a plate with a crack of a finite length $2 a^{\prime}$ and from $\sigma_{c}$, then

$$
d_{3}=2 a^{\prime} \frac{\left(q^{\prime} / \sigma_{c}\right)^{2}}{1-\left(q^{\prime} / \sigma_{c}\right)^{2}}
$$

It follows from (13), (21), that ASFC and FCFC contain the equal characteristic length parameters $d_{1}=d_{3}$, when they are obtained from the experiments for a plate without and with a crack, and lead to the same functional dependencies (11), (19) of the strength $q$ on the crack length $a$.

The plate strength $q=K_{1 c}^{\infty} / \sqrt{\pi a}$ predicted by the linear fracture mechanics can be also presented in terms of the same non-dimensional variables if we express $a$ in terms of $\eta_{3}$ and $K_{1 c}^{\infty}$ from relations (20), (18):

$$
\frac{q}{\sigma_{c}}=\sqrt{\frac{1-\eta_{3}}{2 \eta_{3}}}
$$

One usually evaluates the critical stress intensity factor $K_{1 c}^{\prime}$, instead of $K_{1 c}^{\infty}$, from the formula $q^{\prime}=K_{1 c}^{\prime} / \sqrt{\pi a^{\prime}}$, applying the test with a crack of a finite length $2 a^{\prime}$. Using (21), 
one can rewrite the linear fracture mechanics strength prediction $q / \sigma_{c}=\left(q^{\prime} / \sigma_{c}\right) \sqrt{a^{\prime} / a}$ in the normalized form and get

$$
\frac{q}{\sigma_{c}}=\sqrt{\frac{1-\eta_{3}}{2 \eta_{3}}} \sqrt{1-\frac{q^{\prime 2}}{\sigma_{c}^{2}}}
$$

instead of (22).

Note that it was supposed in deriving (22) that the ratio of the strength predictions of the linear fracture mechanics and of FCFC tends to unity as $a \rightarrow \infty$. In contrast to that, it was supposed in deriving (23) that this ratio is equal to unity at a finite $a=a^{\prime}$. This explain the difference between (22) and (23).

\subsection{Predicted results and comparison with experimental data}

To represent all results in the same coordinates, let us introduce a common non-dimensional crack length parameter $\eta=a /\left(a+d_{0}\right)$, then

$$
\eta_{i}=\frac{\eta}{\eta+\alpha_{i}(1-\eta)}
$$

where $\alpha_{i}=d_{i} / d_{0}$. In what follows, it is assumed that $d_{0}=d_{1}=d_{3}$ and all plots are presented in the non-dimensional coordinates $\eta=a /\left(a+d_{0}\right)$ and $q / \sigma_{c}$. Then $\eta_{1}=\eta_{3}=\eta$ but $\eta_{2}$ is given by (24) where $\alpha_{2}$ is determined by (17).

Note, that all the non-local fracture criteria give $q / \sigma_{c} \rightarrow 1$ when $\eta \rightarrow 0$ for small cracks, as would be expected, while the linear fracture mechanics criterion gives the unrealistic prediction $q / \sigma_{c} \rightarrow \infty$, as can be seen from (22).

From formulas (11), (15), (17), and (24), one can express the relative difference $\delta_{21}:=$ $\left(q_{2}-q_{1}\right) / q_{1}$ in the prediction of the strength of the plate with a crack between ASFC (coinciding with FCFC) and MSFC. It is always positive and its maximum with respect to $\eta$ is equal to $\delta_{21}^{m}=\left(1-\alpha_{2}\right) / \sqrt{1-2 \alpha_{2}}-1$ and is reached at $\eta=\alpha_{2}^{2} /\left(\alpha_{2}^{2}-3 \alpha_{2}+1\right)$, where $0 \leq \alpha_{2} \leq 1 / 4$ according to (17). Thus the maximum of $\delta_{21}$ with respect to $\alpha_{2}$ and $\eta$ is reached at $\alpha_{2}=1 / 4$ (i.e., at the case when $d_{1}, d_{2}, d_{3}$ are obtained from the two fracture tests: without crack and with the infinitely large crack) at $\eta=0.2$ (i.e., at $\left.a=d_{2}=d_{1} / 4=d_{3} / 4\right)$ and is equal to $\delta_{21}^{*}=(3 \sqrt{2}) / 4-1 \approx 0.06$. It means that if $d_{1}, d_{2}, d_{3}$ are obtained from the fracture test without a crack and from a test with a crack of any length, the maximal relative difference between ASFC/FCFC and MSFC predictions for the cracked plate do not exceed $6 \%$.

Data obtained from experiments for laminated composite plates containing short cracks $[2,4,5]$ were chosen to apply each of the criteria described above. The test result for the crack of maximal length was used to calculate $d_{i}$ for each material by (13), (16), (21). Plots for the three considered criteria, whose parameters are obtained by experiments on the different composites, are shown in Fig.4-8 together with the corresponding experimental points. The dashed line corresponds to the prediction of the linear fracture mechanics (23). All of the experimental data used was taken with an allowance for the finite widths of the specimens.

The use of the standard non-dimensional variables $\eta$ and $\lambda=q / \sigma_{c}$ for the presentation of the obtained results permits the marking of data of several series of experiments for the different materials on the same plot for a chosen fracture criterion. Such common pictures of experimental data in comparison with theoretical predictions of the average stress fracture criterion (coinciding with the fictitious crack criterion) and the minimum 
stress fracture criterion, are given in Fig. 9 and 10, respectively. Obviously, the $\eta-$ normalizations of the experimental points differ for the different materials because of the different values of $d_{i}$ obtained. In addition, the limiting value $1 / 4$ for the factor $\alpha_{2}$ was used for the $\eta$-normalizations of the experimental points in Fig. 10.

As can be seen from these figures, all the fracture criteria overestimate somewhat the plate strength for most short cracks (when $d_{i}$ were determined from the tests with the maximum length cracks).

\section{4}

\section{Strength of the plate containing a circular hole}

Consider a circular hole of a radius $a$ in an isotropic plate of infinite extent. The origin of an $\left(x_{1}, x_{2}\right)$ coordinate system coincides with the center of the hole. The solution of linear theory of elasticity for the plate with a hole is well known (see, e.g., [13,14]). If a uniform tensile stress $\sigma$ is applied parallel to the $x_{2}$ axis at infinity, then the normal stress $\sigma_{22}\left(x_{1}, 0\right)$ along the $x_{1}$ axis $\left(x_{1} \geq a\right)$ is given by the expression:

$$
\frac{\sigma_{22}\left(x_{1}, 0\right)}{\sigma}=1+\frac{1}{2}\left(\frac{a}{x_{1}}\right)^{2}+\frac{3}{2}\left(\frac{a}{x_{1}}\right)^{4}
$$

The stress distribution in the non-dimensional coordinates, as it follows from (25), is independent of hole size and the stress concentration factor at the edge of a hole is independent of hole radius too, $\sigma_{22}(a, 0)=3 \sigma$. However, the size of the stress concentration region depends on the hole radius.

Let us consider the strength predictions for this plate with the smooth concentrator by the three NLFC given above. Let us introduce, as in section 3, the normalized hole radius parameters $\eta_{i}=a /\left(a+d_{i}\right)$ where $d_{i}$ are the characteristic lengths of the corresponding criteria.

\subsection{Average stress fracture criterion (ASFC)}

Substituting the equation (25) into (2) and performing the integration yield the fracture criterion for the most stressed point $(a, 0)$ at the plate with the hole:

$$
\frac{q}{\sigma_{c}}=\frac{2}{\left(1+\eta_{1}\right)\left(2+\eta_{1}^{2}\right)}
$$

One can easily see that for large values of the radius $a$ (i.e., when $\eta_{1} \rightarrow 1$ ), the plate strength reduction caused by the hole is determined by the factor $q / \sigma_{c} \rightarrow 1 / 3$, while for small values of $a$ (i.e., when $\eta \rightarrow 0$ ) no strength reduction is predicted, $q / \sigma_{c} \rightarrow 1$.

\subsection{Minimum stress fracture criterion (MSFC)}

According to criterion (4) and (25), one can obtain the following fracture criterion:

$$
\frac{q}{\sigma_{c}}=\frac{2}{2+\eta_{2}^{2}+3 \eta_{2}^{4}}
$$

For the limiting cases of large holes $\left(\eta_{2} \rightarrow 1\right)$ and small ones $\left(\eta_{2} \rightarrow 0\right)$ it follows from (27) that $q / \sigma_{c} \rightarrow 1 / 3$ and $q / \sigma_{c} \rightarrow 1$, respectively. 


\subsection{Fictitious crack fracture criterion (FCFC)}

Placing a fictitious crack of a length $d_{3}$ at the most stressed point $(a, 0)$ of the plate along the $x_{1}$-axis direction, one obtains the concentrator of a non-symmetric form: a circular hole with a single crack originating at its boundary. The symmetric form with two cracks used earlier $[1,4]$ corresponds to the simultaneous fracture at two points. It seems more natural for us not to demand the fracture symmetry.

A linear elastic solution for the concentrator of such a form was obtained by Hsu $[15,16]$. This solution gives the following expression for the stress intensity factor,

$$
K_{1}=\sigma \sqrt{\pi d_{3}} f\left(\eta_{3}\right)
$$

where the function $f\left(\eta_{3}\right)$ (more exactly, $\tilde{f}(\varepsilon)$ where $\varepsilon=d_{3} / a$ ) is given in [15] in a table form.

In the limiting case, when the hole radius $a$ tends to zero, $\eta_{3} \rightarrow 0$ and $f\left(\eta_{3}\right) \rightarrow 1 / \sqrt{2}$. So, the following fracture criterion takes place for the plate without a hole:

$$
K_{1 c}=\sigma_{c} \sqrt{\pi d_{3} / 2}
$$

The fracture criterion $K_{1}=K_{1 c}$ together with equations (28) and (29) gives:

$$
\frac{q}{\sigma_{c}}=\frac{1}{\sqrt{2} f\left(\eta_{3}\right)} .
$$

Note that for the hole with the radius $a \rightarrow \infty, \quad \eta_{3} \rightarrow 1$ and the limiting geometry corresponds to the half-plane with the edge crack under the tensile load $3 \sigma$. Using the known solution of this problem (see, e.g., [17]), one can calculate the limiting value $\lim _{\eta_{3} \rightarrow 1} f\left(\eta_{3}\right) \simeq 3.3645$.

\subsection{Predicted results and comparison with experimental data}

To compare predictions of all the criteria considered, we will use, as in section 3 , the common normalization $\lambda=q / \sigma_{c}$ and $\eta=a /\left(a+d_{0}\right)$; then $\eta_{i}$ is connected with $\eta$ by (24), where $\alpha_{i}=d_{i} / d_{0}$. It is taken further that $d_{0}=d_{1}$.

It should be noted in advance that the fictitious crack fracture criterion is not correct for holes of large radius, since the reduction of strength described by (30) equals approximately 0.21 in the limiting case $a \rightarrow \infty \quad(\eta \rightarrow 1)$ what is more intensive than $1 / 3$ given by linear elasticity stress concentration factor. However, the value $1 / 3$ is true for large holes, as experiments show.

Let us suppose, that the parameters $\sigma_{c}$ (or $K_{1 c}$ ) and $d_{i}$ for the three fracture criteria considered are obtained from the two fracture tests: for a plate without a hole and for the plate with a hole of a radius $a^{\prime}$. One can then get the normalized hole diameter $\eta_{i}^{\prime}$ from the plate strength $q^{\prime}=q\left(a^{\prime}\right)$ and from formulas (26), (27), (30) for each criterion; the characteristic lengths thus are $d_{i}=\left(a^{\prime} / \eta_{i}^{\prime}\right)-a^{\prime}$.

Let us now compare the maximum relative difference between the strength predictions given by ASFC and MSFC criteria. From (24), (26), (27) we have,

$$
\delta_{21}:=\frac{q_{2}-q_{1}}{q_{1}}=\frac{(1+\eta)\left(2+\eta^{2}\right)}{2+\eta^{2}\left(\eta+\alpha_{2}(1-\eta)\right)^{-2}+3 \eta^{4}\left(\eta+\alpha_{2}(1-\eta)\right)^{-4}}-1 .
$$

Using (26) and (27), we can express $\eta_{2}^{\prime}$ in terms of $\eta_{1}^{\prime}=\eta^{\prime}$ and then, using (24), express $\alpha_{2}$ in terms of $\eta^{\prime}$ :

$$
\alpha_{2}=\frac{\eta^{\prime}}{1-\eta^{\prime}}\left(\sqrt{\frac{6}{-1+\sqrt{12\left(1+\eta^{\prime}\right)\left(2+\eta^{\prime 2}\right)-23}}}-1\right) .
$$


Analysis of this expression shows that $0<\alpha_{2}<1 / 2$ for $0<\eta^{\prime}<1, \alpha_{2} \rightarrow 0$ when $\eta^{\prime} \rightarrow 0$, and $\alpha_{2} \rightarrow 1 / 2$ when $\eta^{\prime} \rightarrow 1$. Analysing then the maximum of expression (31) with respect to $\eta$ and $\alpha_{2}$ such that $0<\eta^{\prime}<1,0<\alpha_{2}<1 / 2$, we obtain that the maximum is reached at $\alpha_{2} \rightarrow 1 / 2$ (i.e., at $a^{\prime} \rightarrow \infty$ ) and at $\eta \approx 0.29888$ (i.e., at $a \approx 0.42629 d_{1} \approx 0.85258 d_{2}$ ) and is equal to $\delta_{21}^{*} \approx 0.15659$. Analogously, the minimum of (31) is reached at $\alpha_{2} \rightarrow 0$ (i.e., at $a^{\prime} \rightarrow 0$ ) and at $\eta \rightarrow 0$ (i.e., at $a \rightarrow 0$ ) and is equal to $\delta_{21}^{* *}=-2 / 3$. It means that if $d_{1}, d_{2}$ are obtained from the two fracture test without and with a circular hole, the MSFC strength predictions can amount from $33.3 \%$ to $115.7 \%$ of the ASFC predictions for the plate with a circular hole.

Comparisons of predicted and experimental [1-3] data for the strength of multi-layer composite materials are given in the Fig. 11-17. The characteristic lengths $d_{i}(i=1,2,3)$ were determined for all criteria on the basis of the experiment for the concentrator of the maximal size except for the experiment on the graphite-epoxy laminate with holes of large radius [1] in Fig. 15-17, where the minimal size concentrator was used. The dashed line corresponds to the strength evaluation on the basis of elastic stress concentration factor.

As in the corresponding pictures of section 3 , the $\eta$-normalizations of the experimental points on Fig. 15-17, including the common set of experimental data, differ for the different materials because of the different values of $d_{i}$. In addition, the values $\alpha_{2}=1 / 4$ and $\alpha_{3}=1$ were used for the $\eta$-normalizations of the experimental points in Fig. 16-17.

\section{5}

\section{Some remarks about the fictitious crack fracture criteria}

In addition to the remark given in the point 4.4, some more serious criticism of the fictitious crack fracture criterion can be given.

Let us consider a rectilinear elastic plate under the action of a uniform tensile tractions $q$ applied to two of its sides, Fig. 18. Placing the fictitious crack parallel to the loaded sides of the plate at a distance $h$ from one of these sides, one can see (e.g., from [17]) that the stress intensity factors $K_{1}$ at the crack ends tend to infinity, when $h$ tends to zero. It means, that for any arbitrarily small $q$ there exists sufficiently a small distance $h$ such that FCFC will predict the fracture, what leads to a contradiction with experimental data and experience.

In defence of this criterion, one can say that the type of the boundary conditions such as positive tractions prescribed, is only a mathematical (or mechanical) model and does not exist in reality. But the rejection of this model seems to be too heavy sacrifice for saving the criterion.

\section{Conclusion}

The following conclusions may be drawn on the basis of the performed analysis.

1. All the non-local fracture criteria considered, allow to describe the strength of bodies containing smooth concentrators as well as singular ones. It was shown that the fictitious crack fracture criterion coincides completely with the average stress criterion for the plate with a central crack under symmetric loading.

2. Both the average stress fracture criterion and the minimum stress fracture criterion describe the dependence between body strength and the size of concentrator qualitatively correct. The fictitious crack fracture criterion gives incorrect results for large smooth concentrators and does not enable the limiting transition to the 
local strength condition for the half-plane. In addition, it gives extremely incorrect results even for the uniform stress state under boundary tractions.

3. The best description of experimental data was obtained on the basis of average stress fracture criterion.

4. The considered criteria containing two material parameters do not enable the known experimental data on the strength of bodies with small concentrators to be described exactly. There are systematic deviations of the predicted result from the experimental data. Thus, the the non-local fracture criteria considered need to be improved.

\section{Acknowledgements}

This research was completed while the authors were visiting at the University of Stuttgart, Germany, under support of the project "Application of a functional approach to non-local strength conditions and fracture criteria" from the Volkswagen Foundation.

\section{References}

1. Waddoups,M.E.; Eisenmann,J.R.; Kaminski,B.E.: Macroscopic fracture mechanics of advanced composite materials. J. Comp. Materials 5 (1971) 446-454.

2. Whitney,J.M.; Nuismer,R.J.: Stress fracture criteria for laminated composites containing stress concentrations. J. Comp. Materials 8 (1974) 253-265.

3. Pipes,B.R.; Wetherhold,R.C.; Gillespire,J.M.Jr.: Notched strength of composite materials. J. Comp. Materials 13 (1979) 148-160.

4. Pipes,B.R.; Wetherhold,R.C.; Gillespire,J.M.Jr.: Macroscopic fracture of fibrous composites. Mater. Sci. Eng. 45 (1980) 247-253.

5. Awerbuch,J.; Hahn, H.T.: Crack-tip damage and fracture toughness of boron/aluminum composites. J. Comp. Materials 13 (1979)82-107.

6. Mikhailov,S.E.: A functional approach to non-local strength conditions and fracture criteria - I. Body and point fracture. Engng Fracture Mech., 52 (1995) 731-743.

7. Mikhailov,S.E.: A functional approach to non-local strength conditions and fracture criteria - II. Discrete fracture. Engng Fracture Mech., 52 (1995) 745-754.

8. Mikhailov,S.E.: On a functional description of non-local strength and fracture. Existence and uniqueness. In: Mechanisms and Mechanics of Damage and Failure - Proc.of the 11th Europ. Conf. of Fracture. Poitiers-Futuroscope, France, 1996, Vol.1, pp. 195200 .

9. Neuber,H.: Kerbspannungslehre. Springer, Berlin 1937.

10. Novozhilov,V.V.: On necessary and sufficient criterion of brittle strength. Appl. Math. Mech. (PMM) 33 (1969) 212-222.

11. Cruse,T.A.: Tensile strength of notched composites. J. Comp. Materials 7 (1973) 218-229.

12. Caprino,G.; Halpin,J.C.; Nicolais,L.: Fracture mechanics in composite materials. Composites 10 (1979) 223-227. 
13. Muskhelishvili,N.I.: Some basic problems of mathematical theory of elasticity. Groningen, Noordhoff 1953.

14. Savin,G.N.: Stress concentration around holes. Oxford: Pergamon Pr., 1961.

15. Hsu,Y.C.: The infinite sheet with cracked cylindrical hole under inclined tension or in-plane shear. Int. J. Fract. 11 (1975) 571-581.

16. Hsu,Y.C.: The infinite sheet with two radial cracks from cylindrical hole under inclined tension or in-plane shear. Int. J. Fract. 13 (1977) 839-845 (1977).

17. Murakami, Y. (ed.) Stress intensity factors handbook: Volume 1, Oxford: Pergamon Pr. 1987. 


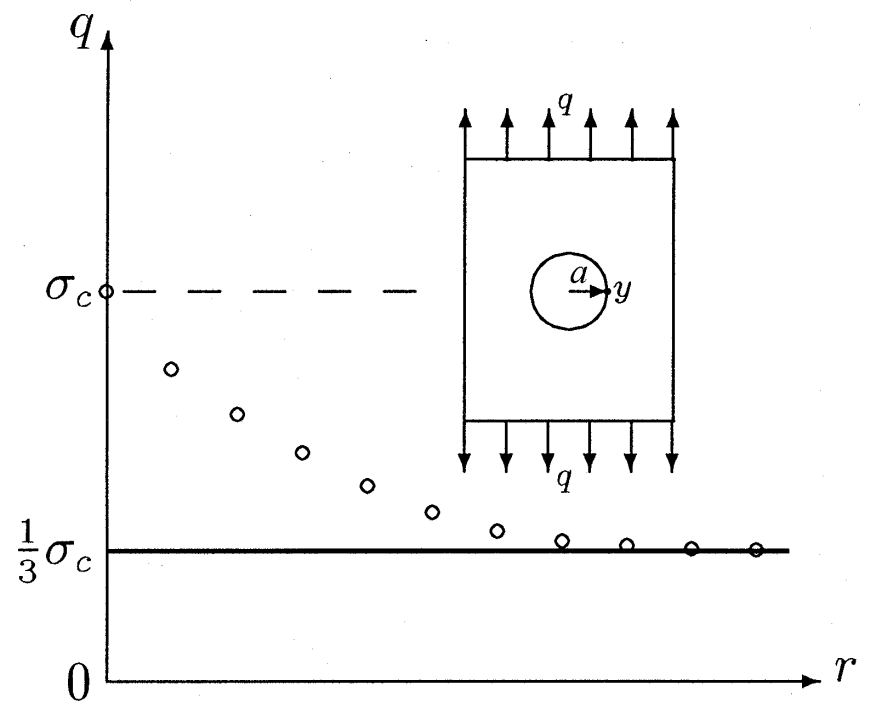

Figure 1. Dependence of the plate strength $q$ on the hole radius $r$ 


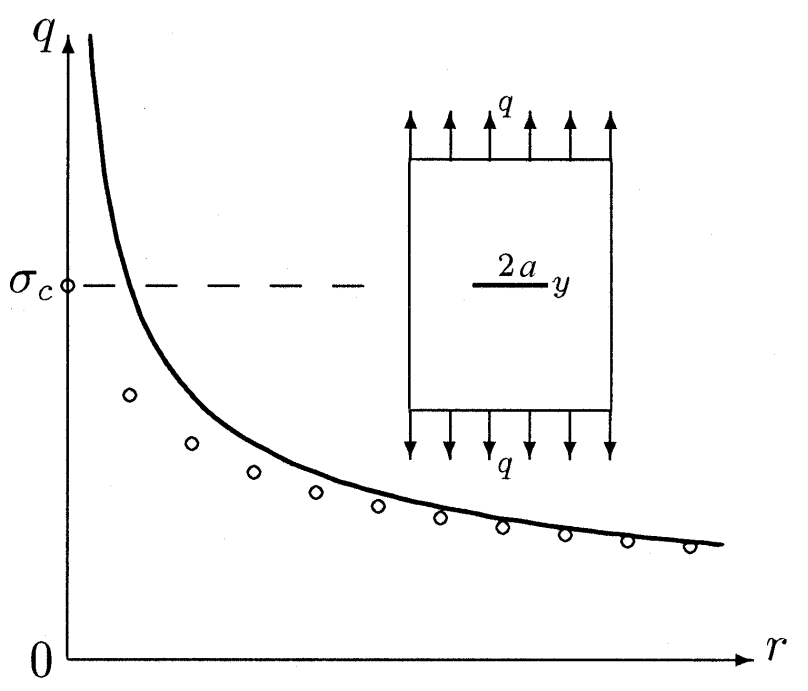

Figure 2. Dependence of the plate strength $q$ on the crack length $r$ 


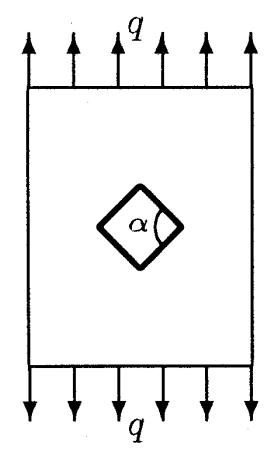

$\sigma_{i j}(\rho, \theta) \sim K_{1}(y ; \alpha) \rho^{-\gamma(\alpha)} f_{i j}(\theta ; \alpha)$

Figure 3. Plate with a diamondshaped hole 


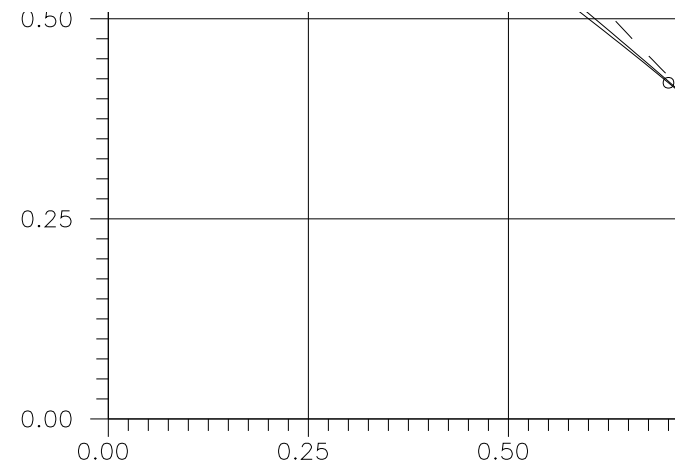

Figure 4. Comparison of predicted and experimental results:

1,3-ASFC and FCFC; 2-MSFC; 4-LFMC;

- experimental data for a $[0 /+45 /-45]_{2 s}$ graphite-epoxy laminate [2]. 


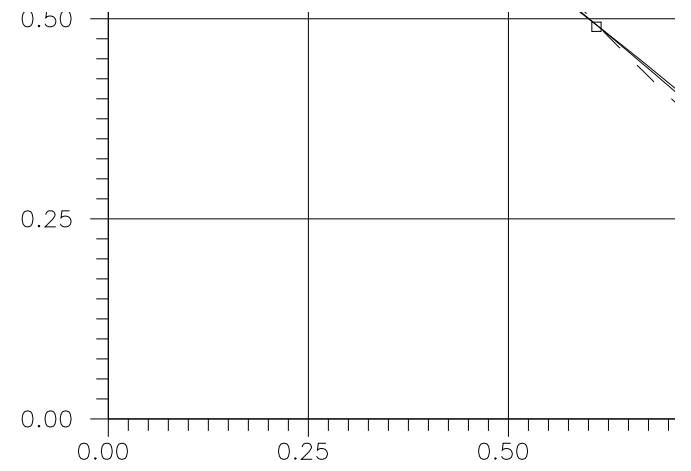

Figure 5. Comparison of predicted and experimental results:

1,3-ASFC and FCFC; 2-MSFC; 4-LFMC;

$\square$ - experimental data for a $[+45 /-45 / 0 / 90]_{s}$ graphite-epoxy laminate [4]. 


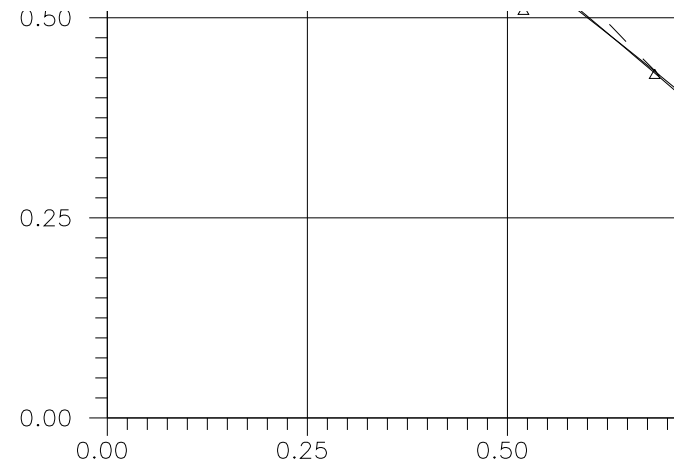

Figure 6. Comparison of predicted and experimental results:

1,3-ASFC and FCFC; 2-MSFC; 4-LFMC;

$\triangle$ - experimental data for a $[90 / 0 /+45 /-45]_{s}$ graphite-epoxy laminate [4]. 


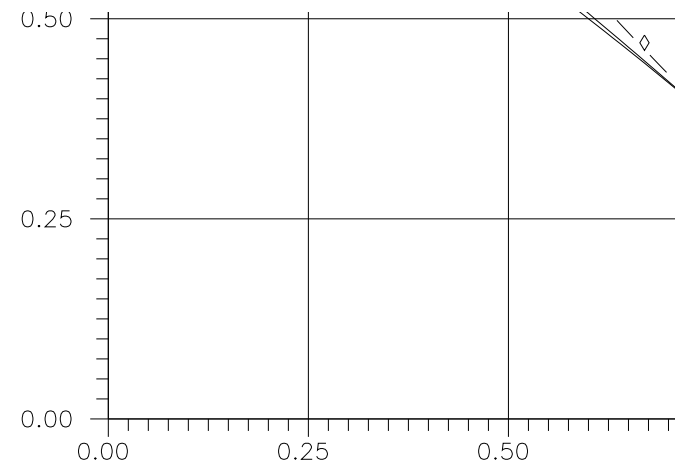

Figure 7. Comparison of predicted and experimental results:

1,3-ASFC and FCFC; 2-MSFC; 4-LFMC;

$\diamond$ - experimental data for a $[0 /+45 /-45 / 90]_{s}$ glass-epoxy laminate [4]. 


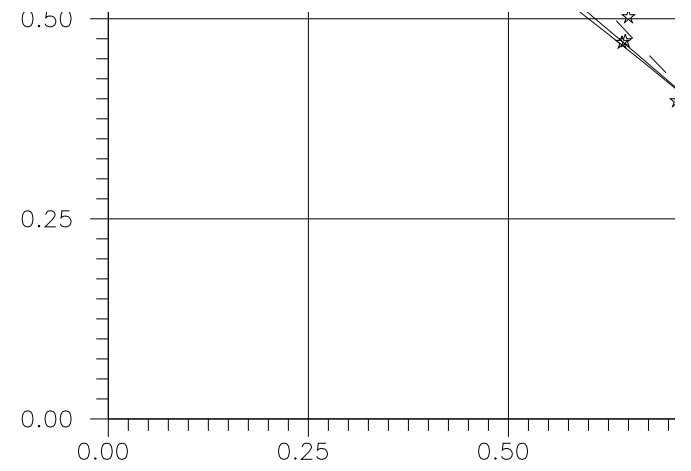

Figure 8. Comparison of predicted and experimental results:

$1,3-A S F C$ and FCFC; 2-MSFC; $4-L F M C$;

* - experimental data for a fibrous composite $A l / B$ [5]. 


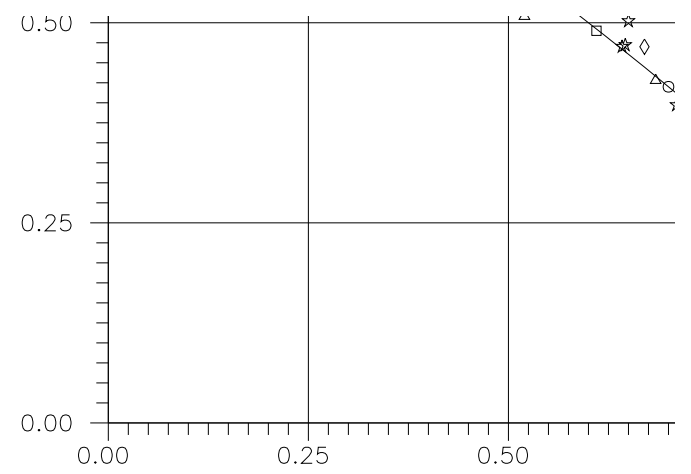

Figure 9. Description of common set of experimental data on the basis of ASFC/FCFC. Marks of experimental points are the same as in Fig. 4-8. 


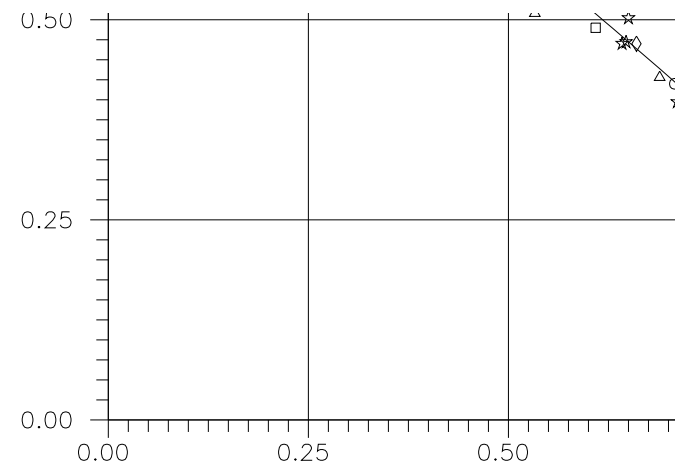

Figure 10. Description of common set of experimental data on the basis of MSFC. Marks of experimental points are the same as in Fig. 4-8. 


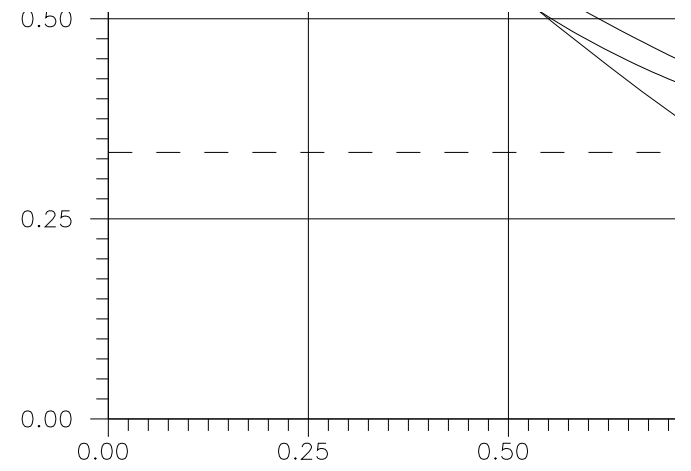

Figure 11. Comparison of predicted and experimental results: 1 - ASFC; 2 - MSFC; 3 - FCFC;

○ - experimental data for a $[0 /+45 /-45]_{2 s}$ graphite-epoxy laminate [1]. 


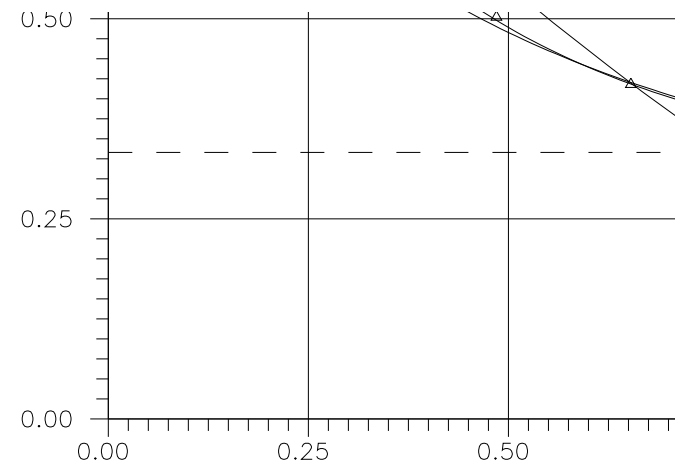

Figure 12. Comparison of predicted and experimental results:

$$
1-A S F C ; \quad 2-M S F C ; \quad 3-F C F C ;
$$

$\triangle$ - experimental data for a quasi-isotropic glass-epoxy composite [2]. 


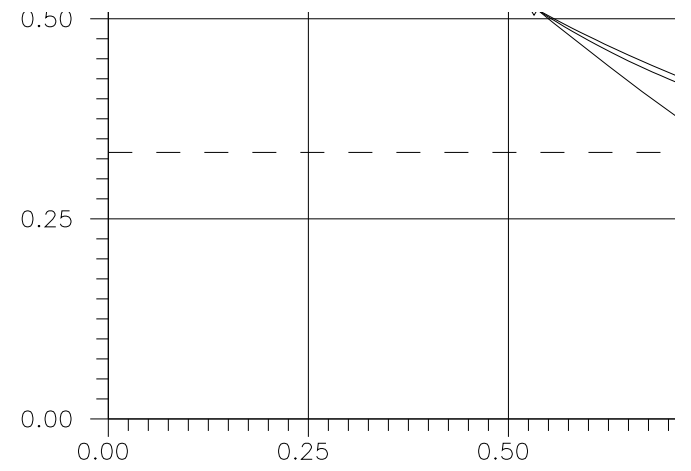

Figure 13. Comparison of predicted and experimental results:

$$
1-A S F C ; \quad 2-M S F C ; \quad 3-F C F C \text {; }
$$

$\diamond$ - experimental data for a $[+45 /-45 / 0 / 90]_{s}$ graphite-epoxy laminate [3]. 


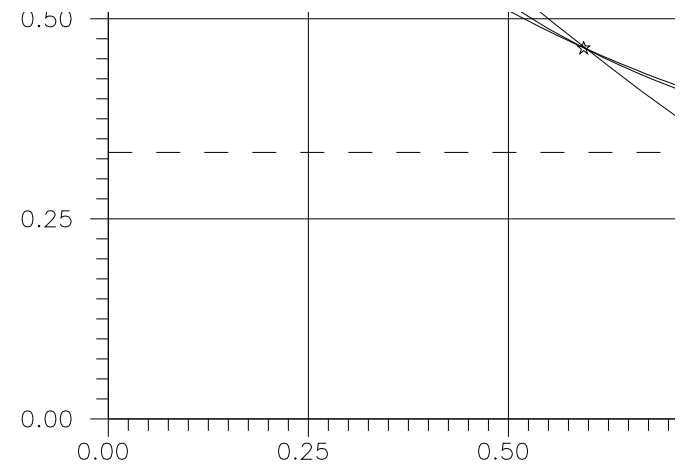

Figure 14. Comparison of predicted and experimental results . $1-A S F C ; \quad 2-M S F C ; \quad 3-F C F C$;

$\star$ - experimental data for a $[90 / 0 /+45 /-45 /]_{s}$ graphite-epoxy laminate [3]. 


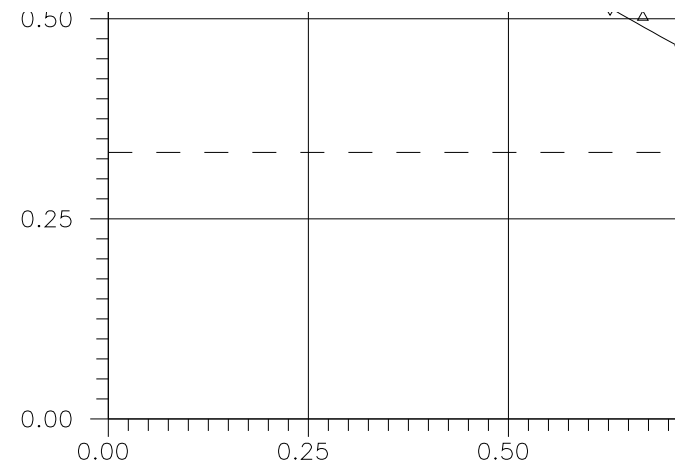

Figure 15.Description of common set of experimental data on the basis of ASFC. Marks of experimental points are the same as in Fig. 11-14;

$\square$ - experimental data for the graphite-epoxy laminate with holes of large radius [1]. 


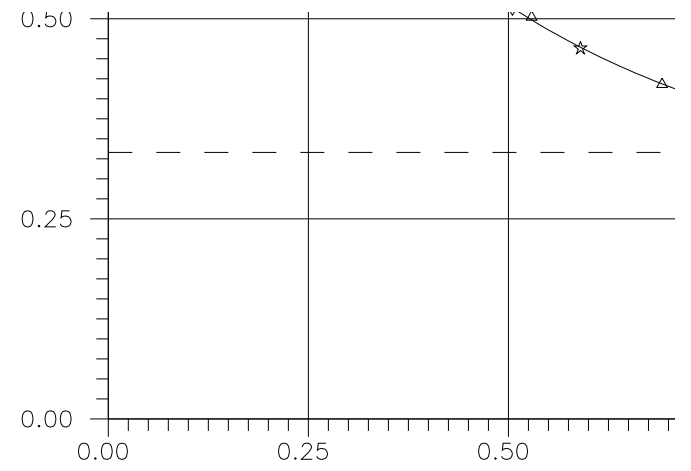

Figure 16. Description of common set of experimental data on the basis of MSFC. Marks of experimental points are the same as in Fig. 13-16;

$\square$ - experimental data for the graphite-epoxy laminate with holes of large radius [1]. 


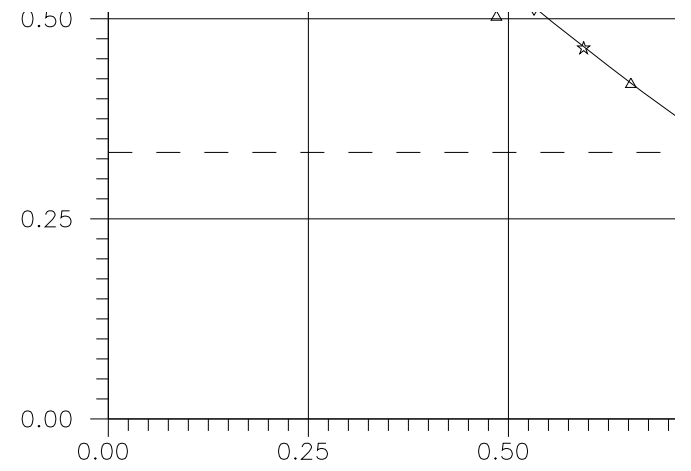

Figure 17. Description of common set of experimental data on the basis of FCFC. Marks of experimental points are the same as in Fig. 13-16;

$\square$ - experimental data for the graphite-epoxy laminate with holes of large radius [1]. 


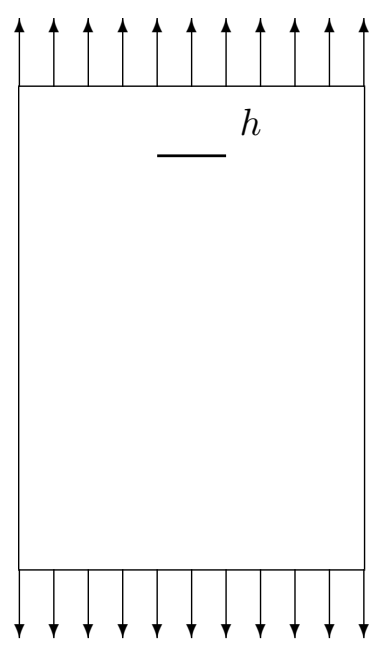

Figure 18. A crack near the plate side loaded by tractions. 